\title{
Differentially-rotating neutron star models with a parametrized rotation profile
}

\author{
F. Galeazzi ${ }^{1}$, S. Yoshida ${ }^{2}$, and Y. Eriguchi ${ }^{2}$ \\ ${ }^{1}$ Max-Planck-Institut für Gravitationsphysik, Albert-Einstein-Institut, Am Mühlenberg 1, 14476 Potsdam, Germany
e-mail: filippo.galeazzi@aei .mpg.de
2 Department of Earth Science and Astronomy, Graduate School of Arts and Sciences, University of Tokyo, Komaba,
Meguro-ku 3-8-1, 153-8902 Tokyo, Japan \\ Recieved 14 December 2010 / Accepted 23 March 2012
}

\section{ABSTRACT}

\begin{abstract}
We analyze the impact of the rotation law on equilibrium sequences of relativistic differentially-rotating neutron stars in axisymmetry. The maximum allowed mass for each model is strongly affected by the distribution of the angular velocity in the radial direction and by the consequent degree of differential rotation. To study the wide parameter space implied by the choice of the rotation law, we introduce a functional form that generalizes the so-called "j-const. law" adopted in all previous work. We compute equilibrium sequences of differentially rotating stars with a polytropic equation of state starting from the spherically symmetric static case. By analyzing the sequences with decreasing degrees of differential rotation, we find that the maximum value of the ratio $T /|W|$ of rotational kinetic energy to gravitational binding energy, is substantially limited in these cases to a value of 0.128 .
\end{abstract}

Key words. gravitation - stars: neutron - stars: rotation - stars: interiors

\section{Introduction}

A neutron star (NS), during most of its life, is considered to be a stationary and rigidly rotating object, apart from a tiny lag between the rotation of the superfluid component and that of the normal fluid and the crust.

A nascent neutron star born in a supernova event is likely to rotate differentially at first and its angular velocity distribution evolves toward a uniform rotation. There are several mechanisms that account for the redistribution of the angular momentum. One such mechanism is the effect of the shear viscosity of neutron matter (Sawyer 1989). An estimate of the timescale on which the viscosity dampens a neutron star's internal shear motion has received a lot of attention in the literature (e.g. Cutler \& Lindblom 1987). For a typical neutron star, we expect a timescale of $10^{1}-10^{2} \mathrm{yr}$. In the presence of magnetic fields, the magnetic braking (Spruit 1999) or the magnetorotational instability (MRI) (Balbus \& Hawley 1991) may drastically accelerate the redistribution of angular momentum to the order of 1s (Duez et al. 2006).

Differential rotation plays an important role at both the beginning and the end of the life of a NS. In its early life, the strong differential rotation of the massive core of a supernova may affect the collapse and bounce dynamics (Dimmelmeier et al. 2002; Ott et al. 2004). A newly born neutron star with strong differential rotation may lose its stability in the course of the rotational (and thermal) evolution, and may collapse to a black hole or another type of compact star (quark star, hybrid star). This may lead to a unique signal either of neutrinos or gravitational waves from the supernova.

An exciting way in which a neutron star's life can end by spiralling into and consequently merging with a binary companion. Numerical relativistic simulations of neutron star binary mergers (e.g., Ruffert et al. 1996; Ruffert \& Janka 2001; Shibata \& Uryū 2002; Shibata \& Taniguchi 2006; Anderson et al. 2008; Baiotti et al. 2008; Giacomazzo et al. 2011) have shown that there are cases in which the remnant of the merger has a significantly high degree of differential rotation such that it can sustain a total mass considerably higher than that of a uniformly rotating star (Baiotti et al. 2008). These hyper-massive neutron stars (HMNSs) remain stable over many dynamical timescales before collapsing to form a black hole (BH) (e.g. Shibata \& Uryū 2002; Baiotti et al. 2008). To study the effect of the centrifugal force in supporting the HMNS, most of these papers show the angular velocity profile of the merged object before the eventual collapse to form a BH. The typical profile extracted from the simulations characteristically follows a plateau near the rotation axis and at a certain distance from this has a nearly power-law behavior. The power index seems to differ from simulation to simulation, but generally does not agree with the so-called "j-const. law" (Eriguchi \& Müller 1985) of rotation, which has so far been the only choice available to construct equilibrium models of differentially rotating stars.

Models of rapidly rotating stars in general relativity have been studied since the 1970s, as large numerical computing facilities became available. The centrifugal deformation and general relativistic gravity make these investigations fully reliant on numerical methods. Since the pioneering work of Butterworth \& Ipser (1976), these studies have included progressively more sophisticated aspects, such as nuclear-matter equation of state (EOS), degrees of differential rotation, magnetic fields, and quite recently meridional flows (Birkl et al. 2011) ${ }^{1}$. However, we note that all of these studies have used just a single kind of ansatz on the rotational angular velocity profile (namely the " $\mathrm{j}$-const. law"), which include uniformly rotating stars as a limiting case (Sect. 2.2).

\footnotetext{
1 We provide examples of references for these studies: Butterworth \& Ipser (1976), Komatsu et al. (1989), Bonazzola et al. (1993), Stergioulas \& Friedman (1995), and Ansorg et al. (2002). For a more comprehensive collection of the literature, see Stergioulas (2003).
} 
In this work, we introduce a new parametrized functional form of the rotation profile that enables us to investigate a broader class of differentially rotating stars. In particular, our new rotation law reproduces different power laws in the outer envelope of the angular velocity distribution (see Sect. 2.2).

The paper is organized as follows. In Sect. 2, we briefly review the formalism of the equations and the numerical method used to solve them. We then describe the new functional form of the rotation profile. In Sect. 3, we construct equilibrium sequences of rotating stars with the new rotation profile. Equilibrium sequences are constructed by imposing a fixed value of $T /|W|$ close to the classical limit for the onset of secular instability; rotating neutron stars are known to be destabilized by the effect of dissipative mechanisms such as viscosity and a gravitational radiation reaction (Chandrasekhar-Friedman-Schutz (CFS) instability; Chandrasekhar 1970; Friedman \& Schutz $1978)$. We show that the maximum value of $T /|W|$ is strongly affected by the rotational angular profile and for a limited class of rotation profiles it is possible to reach a value that exceeds 0.128 . We provide a discussions in our final section.

\section{Formulation}

\subsection{Equations for fluid and spacetime}

We construct configurations for an axisymmetric and stationary rotating perfect fluid in general relativity. The spacetime is assumed to be asymptotically flat and the flow is assumed to be circular (the velocity is only in the azimuthal direction). In this case, we have two Killing vectors (Bardeen 1970) and can choose a coordinate in such a way that the metric of the spacetime is written as (e.g., Komatsu et al. 1989)

$\mathrm{d} s^{2}=-e^{2 v} \mathrm{~d} t^{2}+e^{2 \alpha}\left(\mathrm{d} r^{2}+r^{2} \mathrm{~d} \theta^{2}\right)+e^{2 \beta} r^{2} \sin ^{2} \theta(\mathrm{d} \varphi-\omega \mathrm{d} t)^{2}$,

where the spherical polar coordinates $(r, \theta, \phi)$ are used. The metric potentials $\alpha, \beta, \nu$, and $\omega$ are functions of $r, \theta^{2}$. The energy momentum tensor $T^{a b}$ of a perfect fluid is

$T^{a b}=(\epsilon+p) u^{a} u^{b}+p g^{a b}$

where $\epsilon, p$, and $u^{a}$ are the total energy density, the pressure, and the four velocity, respectively.

The basic equations are: 1) Einstein's equation for the metric potentials $\left.G_{a b}=8 \pi T_{a b} ; 2\right)$ rest mass conservation $\nabla_{a}\left(\rho u^{a}\right)=0$, which is trivially satisfied under the present assumptions; and 3) stress-energy conservation $\nabla_{b} T^{a b}=0$.

As described in Komatsu et al. (1989), the components of Einstein's equation are cast into four equations for the potentials, three of which are elliptic partial differential equations. They are transformed into handy integral equations using the appropriate Green's functions.

The three velocity $V$ of the flow under our current assumptions is given by

$V=e^{\beta-v} r \sin \theta(\Omega-\omega)$,

where $\Omega=u^{\varphi} / u^{t}$ is the angular frequency of the fluid measured in the asymptotic inertial frame. We next introduce the specific angular momentum ${ }^{3} j$

$j=-\frac{u_{\varphi}}{u_{t}}$.

\footnotetext{
$\overline{2}$ We use geometrized units, $c=G=1$.

3 This quantity is one definition of specific angular momentum in general relativity. For different definitions, see Kozlowski et al. (1978).
}

Using these quantities, we can write the equation of hydrostatic equilibrium as

$$
\frac{\partial_{A} p}{\epsilon+p}+\partial_{A} v-\frac{1}{1-V^{2}} V \partial_{A} V+\frac{j}{1-j \Omega} \partial_{A} \Omega=0 \quad(A=r, \theta)
$$

Assuming the fluid to be barotropic, we impose the compatibility of Eq. (5), which enables the equation to be integrated. This condition is written as

$$
\frac{j}{1-j \Omega}=g(\Omega)
$$

by introducing an arbitrary functional $g$, which is detailed in the next subsection. As a result, the first integral of the hydrostatic equation is written as

$$
\int \frac{\mathrm{d} p}{\epsilon+p}+v+\frac{1}{2} \ln \left(1-V^{2}\right)+\int g(\Omega) \mathrm{d} \Omega=0 .
$$

The components of Einstein's equation and the first integral of hydrostatic equation can be iteratively solved as described in Komatsu et al. (1989).

\subsection{Rotation profile}

To exploit the handy first integral of the hydrostatic equation, we need to impose the integrability condition Eq. (6). Different choices of the functional form $g(\Omega)$ would lead to various classes of differential-rotation profiles. All previous studies of differentially rotating relativistic stellar models assumed the simplest linear functional form

$g(\Omega)=A^{2}\left(\Omega_{\mathrm{c}}-\Omega\right)$,

where $A$ is a constant with the dimensions of a length and $\Omega_{\mathrm{c}}$ is the angular velocity on the axis of rotation.

This choice is termed a " $\mathrm{j}$-const. law" since the Newtonian limit of the specific angular momentum $j$ is that of " $\mathrm{j}$-const. law" in Newtonian rotating stars (Eriguchi \& Müller 1985).

We here introduce a more flexible form of the rotation profile as

$g(\Omega)=\frac{\frac{R_{0}^{2}}{\Omega_{\mathrm{c}}^{\alpha}} \Omega\left(\Omega^{\alpha}-\Omega_{\mathrm{c}}^{\alpha}\right)}{1-\frac{R_{0}^{2}}{\Omega_{\mathrm{c}}^{\alpha}} \Omega^{2}\left(\Omega^{\alpha}-\Omega_{\mathrm{c}}^{\alpha}\right)}$,

where $\alpha, R_{0}$, and $\Omega_{\mathrm{c}}$ are constants. The corresponding specific angular momentum is

$j=\frac{R_{0}^{2}}{\Omega_{\mathrm{c}}^{\alpha}} \Omega\left(\Omega^{\alpha}-\Omega_{\mathrm{c}}^{\alpha}\right)$.

The physical significance of the parameters $\alpha, R_{0}$ and $\Omega_{\mathrm{c}}$ in the profile is easily seen if we consider its Newtonian limit. The angular frequency is then written as

$\Omega=\Omega_{\mathrm{c}}\left(1+\left(\frac{R}{R_{0}}\right)^{2}\right)^{\frac{1}{\alpha}}$,

where $R=r \sin \theta$. For $R \ll R_{0}$, we have $\Omega \sim \Omega_{\mathrm{c}}$, while for $R \gg R_{0}$,

$\Omega \sim \Omega_{\mathrm{c}}\left(\frac{R}{R_{0}}\right)^{\frac{2}{\alpha}}$. 
Table 1. Different power-law distributions of the angular velocity in the Newtonian limit for several values of the new rotation law parameter $\alpha$.

\begin{tabular}{lcccc}
\hline \hline$\alpha$ & -1 & $-4 / 3$ & -2 & -4 \\
$\Omega_{\text {outer }}$ & $R^{-2}$ & $R^{-3 / 2}$ & $R^{-1}$ & $R^{-1 / 2}$ \\
Power law in envelope & j-const. & Keplerian & v-const. & HMNS \\
\hline
\end{tabular}

This means that the rotation profile has an inner plateau inside $R \sim R_{0}$ and a power-law envelope for $R \gg R_{0}$. The introduction of the index $\alpha$ is an important feature of the new profile: choosing different $\alpha$, it is possible to describe different power-law distributions of the angular velocity in the outer regions of a star. Table 1 shows the Newtonian limits of particular choices of $\alpha$. The label "j-const." refers to constant specific angular momentum, "v-const." is for constant linear velocity, "HMNS" is the approximate power-law of some numerical relativistic simulations with HMNS formation (e.g., Shibata \& Uryū 2002; Baiotti et al. 2008).

For some interesting cases ( $\alpha \in\{-1,-2,-4\}$ ), it is possible to integrate analytically the expression for $g(\Omega)$ (see Appendix). It is important to note that for $\alpha=-1$ the $j$-constant type of law is recovered - the two functional forms must agree in the limit of weak gravity. This particular case is marginally stable under Rayleigh's local stability criterion of axisymmetric instability, which states that the specific angular momentum must not decrease with increasing radius in a stable star. Values of $\alpha$ smaller than -1 satisfy this condition (and hence are stable), in addition to $\alpha>0$. However star cannot be spun up rapidly enough to allow the appearance of the CFS instability, because the star easily reaches its mass-shedding limit.

We now apply the new rotation law to construct equilibrium configurations of differentially rotating neutron stars and study their dependence on $\alpha$. Each configuration is defined by five parameters: the axis ratio $r_{\mathrm{p}} / r_{\mathrm{e}}$, the polytropic index $N$, the maximum density $\rho_{\max }$, and the two parameters of the rotation law, $R_{0}$ and $\alpha$.

\section{Results}

\subsection{Numerical code}

We construct sequences of axisymmetric and differentially rotating objects using the code discussed in Komatsu et al. (1989). The code iteratively solves Einstein's equation and the first integral of hydrostatic equilibrium.

For all computations, we used a grid with 600 points in the $r$-direction and 300 in the $\theta$-direction. The radial grid was defined in such a way that the inner uniformly-distributed 300 grid points cover the whole star, and on the equator, the 300th point from the origin always corresponds to the surface of the star. Outside the star, we adopt the "compactified" coordinate as in Cook et al. (1992), to take into account the exact boundary condition at the radial infinity.

For a given EOS, a functional of rotation profile and maximum density, the code requires information on "how rapidly" the star is rotating. To this end, it is more convenient to specify a parameter that measures the deformation induced by the rotation rather than the angular momentum or the rotational frequency. We follow Komatsu et al. (1989) in fixing the ratio of the polar coordinate radius of the star to the equatorial radius. The physical parameters of rotation as well as other physical quantities are then computed.

\subsection{Equation of state}

We use a polytropic equation of state, with the index $N=1$ to model neutron stars

$p=\kappa \rho^{1+1 / N}, \epsilon=\rho+N p$.

A typical neutron-star mass and radius are recovered when we set $K=100$ in geometrized units. We fix this parameter for all the sequences to obtain a crude model of nuclear equation of state for neutron matter. In the following paper, we investigate the dependence of the space of configurations on the EOS, using realistic cold and finite-temperature EOSs.

\subsection{Parameters}

To obtain an equilibrium model, we fix the parameters of the rotation profile, $\alpha$ and $R_{0}$, then choose the maximum density $\rho_{\max }$ and the coordinate axis ratio. This set of parameters uniquely determines an equilibrium configuration, whose physical characteristics such as mass, compactness, and angular momentum, angular frequency at the center are computed once the solution is obtained. The rest mass and gravitational mass are computed by using the standard formula (see e.g. Bardeen 1970). An important measure of rotation, the $T /|W|$ parameter, is introduced as in Komatsu et al. (1989). This is used in both Newtonian and general relativistic studies of rotating stars and defined as the ratio of rotational kinetic energy to gravitational binding energy (see Komatsu et al. 1989, for details). It characterises the overall strength of the rotation of the star. Classical studies suggest that when $T /|W| \sim 0.14$, bar-shaped equilibria bifurcate (into "Jacobi" or "Dedekind" type equilibrium sequences). In full general relativity, Stergioulas \& Friedman (1998) showed that the value for the onset of the secular instability is much lower - around half of the Newtonian result. The impact of differential rotation was studied by both Yoshida et al. (2002) and Krüger et al. (2010) for a "j-const". law (in Cowling approximation); instability was found to occur for a larger $T /|W|$ than those found for uniformly rotating stars. From Table 2 of Yoshida et al. (2002), we take as our reference critical value $T /|W|=0.125$. This is the value for an $N=1$ polytropic EOS, where the coefficient $A$ from Eq. (8) is set to unity.

\subsection{Numerical equilibrium sequences}

For a given rotation profile and a maximum density, we start from a non-rotating model (the polar-to-equatorial axis ratio is equal to unity), and decrease the ratio to obtain stars with increasingly rapid rotation. We terminate the sequence if one of the following conditions is satisfied: 1 ) $T /|W|=0.125$, which is our reference value for the onset of secular instability (see discussion at the end of the last paragraph). 2) The mass-shedding limit, which is reached when the angular frequency of matter at the equatorial surface comes close to the local Keplerian frequency and a further spin-up of a star leads to the shedding of mass. Beyond this point it is impossible to construct equilibrium configurations. 3) Topology change. When the degree of differential rotation is large, the maximum density may move away from the rotation axis. When the value of the axis ratio reaches zero, the star develops a hole on the rotation axis. The equilibrium sequence may continue beyond this point, but we are not interested in this toroidal shape configuration as a model of a neutron star.

Concerning the last point above, we should note that the structure of the parameter space is quite complex, as analyzed 
by Ansorg et al. (2009). The toroidal and the spheroidal configurations form disjoint families of solutions separated by a mass shedding region. Increasing the degree of differential rotation by reducing the value of the $R_{0}$ parameter, the two families join again in the parameter space. The area of the region separating the two topologically different families depends on the $\rho_{\max }$ of the configuration, becoming smaller as the latter increases. Stated differently, starting from the Newtonian limit and going to the more general relativistic case, it becomes more and more complicated to find a value of $R_{0}$ for which the solution can reach the toroidal family, since the separation increases between the two classes of solutions. We studied the parameter space of the solutions for the three different rotation laws analyzed here, and will present the results on the dependency hidden in the EOS in a future paper.

\subsection{Stellar mass in parameter space}

To visualize our results, we plot the gravitational mass of stellar models in the parameter space. Figure 1 shows the surfaces of equilibrium models embedded in the $R_{0}-\rho_{\max }-M / M_{\text {sun }}$ space, where $M$ is the gravitational mass in unit of solar masses, for three different values of the parameter $\alpha: \alpha=-1$ (top panel), $\alpha=-2$ (middle panel), and $\alpha=-4$ (bottom panel). In order to ensure the same degree of differential rotation for all the models with a constant $R_{0}$, following the prescription of Baumgarte et al. (2000), we define:

$\hat{R}_{0}=R_{0} / R_{\mathrm{c}}$,

where $R_{\mathrm{c}}$ is the circumferential radius.

On the floors of these plots, we marked each parameter pair ( $\left.\rho_{\max }, \hat{R}_{0}\right)$ with different symbols to indicate how the equilibrium sequence ends. The black triangles corresponds to the parameters for which $T /|W|$ of the model reaches 0.125 (and the computations are stopped there). The dots correspond to the parameter pairs for which the sequence terminates at the mass-shedding limit. For small values of $\hat{R}_{0}$, the sequence of spheroids ends with a topological change.

As mentioned in Sect. 3.4, our sequences terminate at one of the three different criteria, thus one needs to be careful in reading these figures. We note that for the sequences terminating at $T /|W|=0.125$ and at the topological change, the value of mass plotted here is not the maximum mass of the sequence. The solutions of the toroidal class do not possess a value for the maximum mass, which can instead increase arbitrarily as the torus becomes increasingly thinner and thinner along the sequence of equilibrium figures (see Ansorg et al. 2003).

It can be seen from Eq. (9) that the angular velocity profile becomes uniform as $\hat{R}_{0} \rightarrow \infty$. In this rigid rotation limit, the sequence of $N=1$ polytropes is known to terminate with a mass-shedding state before reaching $T /|W|=0.125$. The degree of differential rotation depends on both the power-law exponent $\alpha$ and the radius $R_{0}$. We may regard it as weak when the profile is close to uniform rotation (i.e. $R_{0} \rightarrow \infty$ and/or $\alpha \rightarrow-\infty$ ). As in the case of uniform rotation, for stars with weak differential rotation the centrifugal force at the equator on the surface reaches a value at which the star sheds mass before its $T /|W|$ value reaches 0.125 . In this case, all the sequences terminate at massshedding limit before reaching either $T /|W|=0.125$ or the point of topology change. However, a star with sufficiently strong differential rotation can store a large amount of rotational energy deep inside the star and allow the surface angular frequency to be lower than that of the mass-shedding limit. Therefore, a star
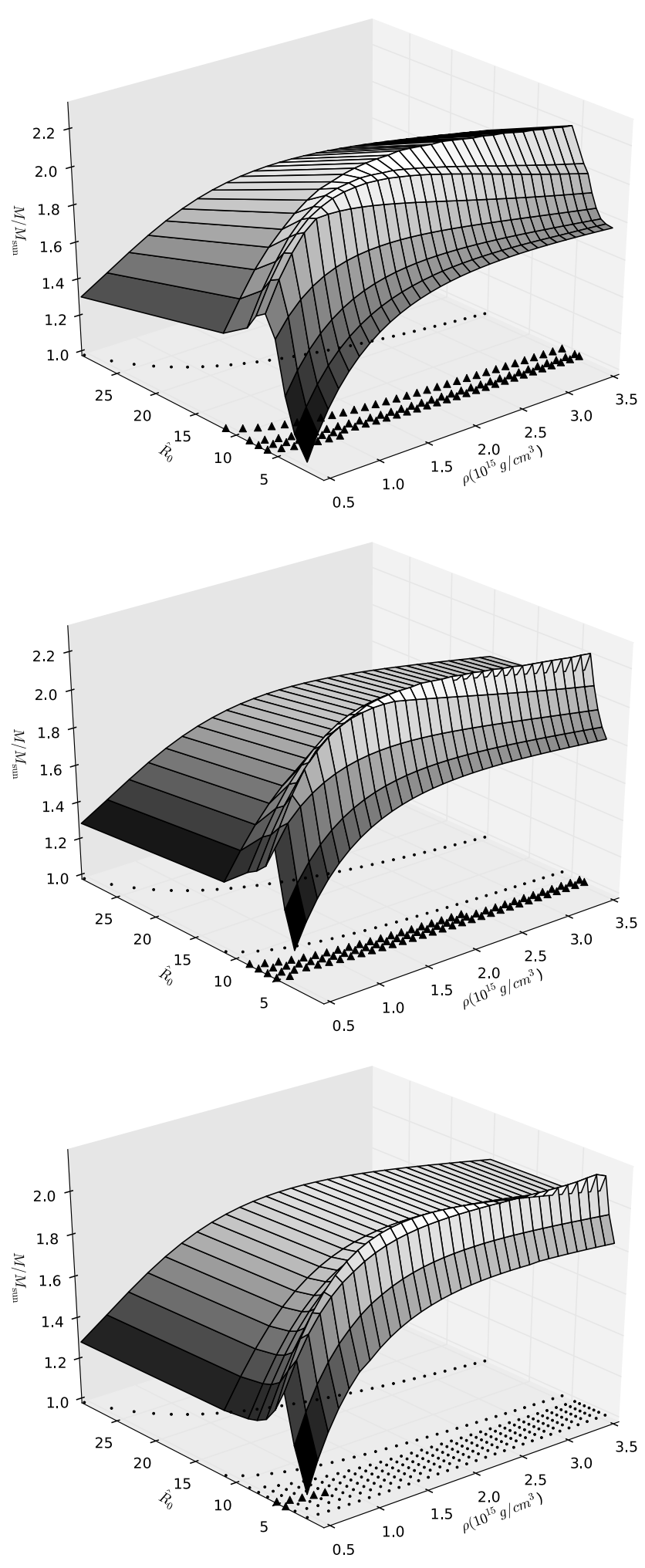

Fig. 1. Two-dimensional surfaces of equilibrium models of differentially rotating neutron stars embedded in the space $\hat{R}_{0}-\rho_{\max }-M / M_{\text {sun }}$. The first panel is for $\alpha=-1$, the second one is for $\alpha=-2$, and the last one is for $\alpha=-4$. These correspond to "j-const.", "v-const.", and "HMNS" rotation laws, respectively. The points at the bottom of each panel are drawn to clarify which of the three terminal conditions (see Sect. 3.4) of sequence applies. The black triangles are for sequences reaching $T /|W|=0.125$, dots are for sequences terminating with mass shedding, and the right edge of each surface (low $\hat{R}_{0}$ ) corresponds to a topology change. The limit $\hat{R}_{0} \rightarrow \infty$ corresponds to uniform rotation. 
with $\alpha=-1$ or -2 reaches the critical point $T /|W|=0.125$ before encountering either mass-shedding or topology change, provided that $\hat{R}_{0}$ is small enough (that is, there is a small enough core region of nearly uniform rotation). For these choices of $\alpha$ and sufficiently small $\hat{R}_{0}$, we also see the appearance of topological change before the critical $T /|W|=0.125$ is reached (represented as the triangles in Fig. 1). The sequences that we terminate at $T /|W|=0.125$ will eventually experience the topology change when extended to faster rotation.

An important observation here is that for $\alpha=-4$ the maximum allowed $T /|W|$ is 0.128 before mass shedding occurs, and for $\alpha=-1$, and -2 we need to set $\hat{R}_{0}$ sufficiently small (i.e., enable a high degree of differential rotation) to achive the critical $T /|W|$ before either topology change or mass shedding occurs.

\section{Summary and discussion}

We have introduced a new rotation profile to study the equilibrium sequences of differentially rotating relativistic stars. Compared with the precedent studies, which assume only one type of rotation profile, we are now able to investigate a broader class of rotating stars.

As a first step of systematic studies, we focus on the simplest neutron-star model with a polytropic EOS with index $N=1$. For the rotation profiles that allow analytic expressions in the first integral of hydrostatic equilibrium equation, we have computed sequences of spheroidal equilibria that start from non-rotating stars. Special attention is paid to the appearance of the critical point signaling the onset of the secular instability (CFS instability) for relativistic differentially rotating stars. We demonstrated that the appearance of the critical point may occur for rather strong differential rotation in the case of $\alpha=-1$ and -2 in Eq. (9), which corresponds respectively to so-called "j-const." and "v-const." rotation profiles in Newtonian stars (Eriguchi \& Müller 1985). Even in these cases, the critical point does not appear before the configuration changes from a spheroidal topology to a toroidal one, unless the parameter $R_{0}$ in Eq. (9) is sufficiently small.

When $\alpha=-4$ instead (which appears to mimic the approximate rotation profile of some of the quasi-stationary HMNSs seen in the numerical simulations of neutron-star mergers), it seems that only a few of the models reaches the critical point of CFS instability before the equilibrium sequences reach their mass-shedding limit. However, these stars may be susceptible to the relatively new class of dynamical instability (so-called "low $T /|W|$ instability", e.g. Centrella et al. 2001; Shibata et al. 2002; Watts et al. 2003; Saijo \& Yoshida 2006; Ou \& Tohline 2006; Baiotti et al. 2008), whose existence relies on the strong shear flow (Watts et al. 2005; Corvino et al. 2010). Numerical simulations of binary NSs do indeed indicate that the HMNS develops a bar deformation (e.g. Shibata \& Uryū 2002; Baiotti et al. 2008) on top of the background star with approximate axisymmetry. It would be interesting to study the appearance of low $T /|W|$ instability by using our models of equilibrium stars with different rotation profiles.

Acknowledgements. We thank Marcus Ansorg and Carlos Palenzuela for useful discussions. This work is supported in part by a Grant-in-Aid for Scientific Research (C) of Japan Society for the Promotion of Science (20540225). F.G. wants to thank Aaryn Tonita for essential support in this work, Sam Lander for fruitful discussions and, finally Luciano Rezzolla for the continuous support that he devoted to this project.

\section{Appendix A: Integration of $g(\Omega)$}

For some interesting cases, we provide an analytic expression for the integral of $g(\Omega)$ that appears in Eq. (7).

We define a parameter $k:=\left(R_{0} \Omega_{\mathrm{c}}\right)^{2}$ and normalize $\Omega$ as $\xi:=$ $\Omega / \Omega_{\mathrm{c}}$. The integration of the functional $g(\Omega)$ is then written as

$\int g(\Omega) \mathrm{d} \Omega=\int \frac{k \xi\left(\xi^{\alpha}-1\right)}{1-k \xi^{2}\left(\xi^{\alpha}-1\right)} \mathrm{d} \xi$.

For $\alpha=-1$, the right hand side reduces to

$\sqrt{\frac{k}{4-k}} \operatorname{Arctan}\left(\sqrt{\frac{k}{4-k}}(2 \xi-1)\right)-\frac{1}{2} \ln (1+k \xi(\xi-1))$.

For $\alpha=-2$, it reduces to

$\frac{\ln \left(1+k\left(\xi^{2}-1\right)\right)-2 k \ln \xi}{2(k-1)}$.

Finally, for $\alpha=-4$, we have

$-\ln \xi+\frac{1}{2 \sqrt{1+4 k^{2}}} \ln \left(\frac{-1+\sqrt{1+4 k^{2}}-2 k \xi^{2}}{+1+\sqrt{1+4 k^{2}}+2 k \xi^{2}}\right)$.

\section{References}

Anderson, M., Hirschmann, E. W., Lehner, L., et al. 2008, Phys. Rev. Lett., 100, 191101

Ansorg, M., Kleinwächter, A., \& Meinel, R. 2002, A\&A, 381, L49

Ansorg, M., Kleinwächter, A., \& Meinel, R. 2003, ApJ, 582, L87

Ansorg, M., Gondek-Rosińska, D., \& Villain, L. 2009, MNRAS, 396, 2359

Baiotti, L., Giacomazzo, B., \& Rezzolla, L. 2008, Phys. Rev. D, 78, 084033

Balbus, S. A., \& Hawley, J. F. 1991, ApJ, 376, 214

Bardeen, J. M. 1970, ApJ, 162, 71

Baumgarte, T. W., Shapiro, S. L., \& Shibata, M. 2000, ApJ, 528, L29

Birkl, R., Stergioulas, N., \& Muller, E. 2011, Phys. Rev. D, 84, 023003

Bonazzola, S., Gourgoulhon, E., Salgado, M., \& Marck, J. A. 1993, A\&A, 278, 421

Butterworth, E. M., \& Ipser, J. R. 1976, ApJ, 204, 200

Centrella, J. M., New, K. C. B., Lowe, L. L., \& Brown, J. D. 2001, ApJ, 550, L193

Chandrasekhar, S. 1970, Phys. Rev. Lett., 24, 611

Cook, G. B., Shapiro, S. L., \& Teukolsky, S. A. 1992, ApJ, 398, 203

Corvino, G., Rezzolla, L., Bernuzzi, S., De Pietri, R., \& Giacomazzo, B. 2010 Class. Quant. Grav., 27, 114104

Cutler, C., \& Lindblom, L. 1987, ApJ, 314, 234

Dimmelmeier, H., Font, J. A., \& Müller, E. 2002, A\&A, 393, 523

Duez, M. D., Liu, Y. T., Shapiro, S. L., Shibata, M., \& Stephens, B. C. 2006, Phys. Rev. D, 73, 104015

Eriguchi, Y., \& Müller, E. 1985, A\&A, 146, 260

Friedman, J. L., \& Schutz, B. F. 1978, ApJ, 222, 281

Giacomazzo, B., Rezzolla, L., \& Baiotti, L. 2011, Phys. Rev. D, 83, 044014

Komatsu, H., Eriguchi, Y., \& Hachisu, I. 1989, MNRAS, 237, 355

Kozlowski, M., Jaroszynski, M., \& Abramowicz, M. A. 1978, A\&A, 63, 209

Krüger, C., Gaertig, E., \& Kokkotas, K. D. 2010, Phys. Rev. D, 81, 084019

Ott, C. D., Burrows, A., Livne, E., \& Walder, R. 2004, ApJ, 600, 834

Ou, S., \& Tohline, J. E. 2006, ApJ, 651, 1068

Ruffert, M., \& Janka, H. 2001, A\&A, 380, 544

Ruffert, M., Janka, H., \& Schaefer, G. 1996, A\&A, 311, 532

Saijo, M., \& Yoshida, S. 2006, MNRAS, 368, 1429

Sawyer, R. F. 1989, Phys. Rev. D, 39, 3804

Shibata, M., \& Taniguchi, K. 2006, Phys. Rev. D, 73, 064027

Shibata, M., \& Uryū, K. 2002, Progr. Theoret. Phys., 107, 265

Shibata, M., Karino, S., \& Eriguchi, Y. 2002, MNRAS, 334, L27

Spruit, H. C. 1999, A\&A, 349, 189

Stergioulas, N. 2003, Liv. Rev. Rel., 6, 3

Stergioulas, N., \& Friedman, J. 1995, AJ, 444, 306

Stergioulas, N., \& Friedman, J. L. 1998, ApJ, 492, 301

Watts, A. L., Andersson, N., Beyer, H., \& Schutz, B. F. 2003, MNRAS, 342 1156

Watts, A. L., Andersson, N., \& Jones, D. I. 2005, ApJ, 618, L37

Yoshida, S., Rezzolla, L., Karino, S., \& Eriguchi, Y. 2002, ApJ, 568, L41 